\title{
Evolución de la serie Microphyllae (Adesmia, Fabaceae) en la Cordillera de los Andes: una perspectiva biogeográfica
}

\author{
Evolution of the series Microphyllae (Adesmia, Fabaceae) in the Andean Cordillera: \\ a biogeographic approach
}

\author{
MARITZA A. K. MIHOČ ${ }^{*}$, JUAN J. MORRONE ${ }^{2}$, MARIA A. NEGRITTO ${ }^{1} \&$ LOHENGRIN A. CAVIERES $^{1,3}$ \\ ${ }^{1}$ Departamento de Botánica, Facultad de Ciencias Naturales y Oceanográficas, Universidad de Concepción, Casilla 160-C, \\ Concepción, Chile \\ ${ }^{2}$ Departamento de Biología Evolutiva, Facultad de Ciencias, Universidad Nacional Autónoma de México (UNAM), \\ Apartado Postal 70-399, 04510 México Distrito Federal, México \\ ${ }^{3}$ Instituto de Ecología y Biodiversidad, Casilla 653, Santiago Chile; \\ *e-mail correspondencia: mmihoc@udec.cl
}

\begin{abstract}
RESUMEN
Microphyllae (subgénero Acanthadesmia) es la serie que posee el mayor número de especies descritas en el género Adesmia, y su distribución incluye la mayor parte del área de distribución del género. El escenario biogeográfico evolutivo, tanto del género como de la serie, es poco claro. Algunas hipótesis sugieren que el desarrollo de los Andes estaría relacionado con la evolución de Adesmia, sin embargo, no se ha establecido el proceso (e.g., dispersión o vicarianza) subyacente a dicha evolución. En este estudio se empleó la aproximación panbiogeográfica mediante un análisis de trazos y un análisis de parsimonia de endemismos (PAE), basados en la distribución de 22 de las especies de esta serie. El área de distribución de la serie fue dividida en cuadrículas de $1^{\circ} \times 1^{\circ}$ (Latitud x Longitud), y franjas latitudinales y longitudinales de $1^{\circ}$. Todas las especies presentaron una distribución asociada con los Andes y restringida tanto latitudinal como longitudinalmente. Encontramos cinco trazos generalizados y dos nodos. Todos los trazos generalizados se asociaron longitudinalmente con la cordillera y los nodos se asociaron con la Zona de Transición Sudamericana. El PAE realizado con las franjas latitudinales establece que el área de distribución de la serie se divide en una zona norte y una sur, cuyo límite se encontraría en Chile central (ca. $33^{\circ} \mathrm{S}$ ). Esta división se encuentra caracterizada por la presencia de $A$. miraflorensis en la zona norte y A. volckmannii en la zona sur. Nuestros resultados sugieren una diferenciación reciente, ocasionada por el aislamiento de poblaciones a lo largo de la cordillera; es decir, eventos vicariantes que fragmentaron la distribución y posteriormente indujeron la diferenciación de grupos. La separación se habría dado en sentido latitudinal, como lo sugiere la secuencia latitudinal de trazos generalizados.
\end{abstract}

Palabras clave: panbiogeografía, análisis de trazos, Andes, Adesmia, PAE.

\begin{abstract}
Microphyllae (subgenus Acanthadesmia) is the series of the genus Adesmia with the largest number of described species, and its distribution includes most of the genus range. The biogeographic evolutionary history of this series, as well as the genus, is unclear. Some hypotheses suggest that the development of the Andes affected the evolution of the genus Adesmia, but there is no evidence about the processes (e.g., dispersal or vicariance) involved. In this study we used a panbiogeographic approach, and based on the distribution of 22 species of this series we carried out a track analysis and a parsimony analysis of endemicity (PAE). Distribution area of the series was divided in quadrats of $1^{\circ} \times 1^{\circ}$ (Latitude x Longitude), and latitudinal and longitudinal belts of $1^{\circ}$. All the species presented a distribution associated with the Andes, and most of them presented both latitudinally and longitudinally restricted distributions. Five generalized tracks and two nodes were found. All tracks were longitudinally associated with the Andean range. Nodes could be associated with the South American Transition Zone. PAE based on latitudinal belts split the total area in two sub-areas (north and south), where the limit between them is central Chile (ca. $33^{\circ} \mathrm{S}$ ). This division is characterized by the presence of A. miraflorensis in the northern zone, and A. volckmannii in the southern zone. Our results suggest a recent differentiation within the series Microphyllae, which may have resulted from isolation of populations along the Andes, i.e., vicariance events fragmented the distribution of species and subsequently caused differentiation. It seems likely that this separation has occurred latitudinally, as suggested by the latitudinal sequence of generalized tracks.
\end{abstract}

Key words: panbiogeography, track analysis, Andes, Adesmia, PAE. 


\section{INTRODUCCIÓN}

Distintos eventos geológicos y climáticos han determinado la estructura y distribución de las comunidades del extremo sur del continente americano, atribuyéndoseles un papel causal en lo que se refiere a extinción, diferenciación y cambios en la distribución geográfica de la biota (Simpson 1983, Nores \& Cerrana 1990, Crisci et al. 1991, Hernández et al. 1992, Hinojosa \& Villagrán 1997, Villagrán \& Hinojosa 1997, Young et al. 2002). Algunos autores han asociado el levantamiento de los Andes con la expansión de la distribución de algunos taxones (Van Der Hammen \& Cleef 1983, Ezcurra et al. 1997). Por ejemplo, varios taxones subantárticos (e.g., Colobanthus Bartl., Cotula L., Oreobolus R. Br., Uncinia Pers. y muchas briófitas) habrían alcanzando el extremo norte de los Andes, dispersándose a lo largo de la zona altoandina (Van Der Hammen \& Cleef 1983). Otros autores, en cambio, han considerado al levantamiento andino como un evento vicariante, que habría dividido al continente en dos áreas: una occidental, con taxones usualmente asignados a trazos generalizados que conectan esta área con Australia y Nueva Zelanda; y una oriental, con taxones asignados a trazos generalizados que la conectan con los trópicos del Viejo Mundo (Katinas et al. 1999, Morrone 2001a).

En América del Sur, el género Adesmia DC. (Adesmiae, Fabaceae) posee un elevado número de especies. Este género consta de dos subgéneros: Adesmia Burkart, distribuido en la Cordillera de los Andes y en las zonas semiáridas de Argentina y Brasil; y Acanthadesmia Burkart, distribuido principalmente a lo largo de la Cordillera de los Andes (Burkart 1967, Burkart \& Correa 1984, Miotto 1993, Davyt \& Izaguirre 1996, Ulibarri \& Burkart 2000). Burkart (1967) plantea una relación estrecha entre la evolución del género y el desarrollo de la Cordillera de los Andes. El escenario propuesto por este autor está basado en dispersión, en donde el motor de la diferenciación (formación de los centros de polimorfismo) sería la elevación de los Andes. Sin embargo, no estableció ninguna conexión entre la diferenciación de los grupos con los procesos subyacentes a la misma.

En el género Adesmia, la serie Microphyllae Burkart es la que posee el mayor número de especies y la más amplia distribución (Burkart 1967, Ulibarri 1987), sus especies son esencialmente andinas, encontrándose de preferencia por sobre los $1.000 \mathrm{~m}$ de altitud, pudiendo llegar hasta los $5.000 \mathrm{~m}$ (Ulibarri 1987, Zuloaga \& Morrone 1999, C. Marticorena, comunicación personal). Ulibarri (1987) realizó la revisión taxonómica de la serie, planteando una evolución reciente del género además de un activo proceso de especiación entre las especies. A diferencia de Burkart (1967), este autor no estableció hipótesis respecto a la historia biogeográfica del grupo.

En el presente trabajo utilizamos métodos panbiogeográficos para establecer un escenario posible sobre la evolución de la serie Microphyllae del subgénero Acanthadesmia en relación con la Cordillera de los Andes, determinando el proceso (dispersión o vicarianza) que explicaría de manera más adecuada su patrón actual de distribución geográfica.

Desde una perspectiva panbiogeográfica, el trazo individual constituye la representación gráfica de una hipótesis relativa a las relaciones en el espacio en el marco del área donde, y en relación con la cual, el grupo evoluciona (Zunino \& Zullini 2003). Esta hipótesis de homología biogeográfica primaria enfatiza la importancia de la dimensión espacial o geográfica para comprender los patrones y procesos evolutivos (Craw et al. 1999, Morrone 2004 a). A su vez, el análisis de parsimonia de endemismos (PAE: Parsimony Analysis of Endemicity) también puede aplicarse para postular una hipótesis preliminar de relaciones entre áreas, basándose en los patrones de distribución de las especies que en ellas habitan (Luna et al. 1999). Ambos métodos biogeográficos pueden ser utilizados para reconocer elementos bióticos, los cuales resultarían básicamente de procesos de vicarianza y constituirían las unidades biogeográficas para análisis posteriores (Morrone 2001b, 2004a).

\section{MATERIALES Y MÉTODOS}

\section{Serie estudiada}

Las especies de Adesmia se distribuyen a lo largo de la Cordillera de los Andes desde el 
norte del Perú hasta Tierra del Fuego $\left(8-54^{\circ} \mathrm{S}\right)$ y, hacia el este, llegan a zonas semiáridas de la Serra do Mar en Brasil (Burkart 1967, Burkart \& Correa 1984, Miotto 1993, Davyt \& Izaguirre 1996, Ulibarri \& Burkart 2000), distribución que corresponde específicamente con la subregión Chaqueña de la región Neotropical, la Zona de Transición Sudamericana y la región Andina (Morrone 1994a, 1996, 2002, 2004b). Las 240 especies descritas del género se encuentran agrupadas en 43 series, subordinadas a dos subgéneros: el subgénero Adesmia contiene plantas inermes y agrupa a 34 series, mientras que el subgénero Acanthadesmia reúne plantas con espinas y agrupa a las nueve series restantes (Burkart
1967, Ulibarri 1980, 1982, 1987). Se seleccionó a la serie Microphyllae, del subgénero Acanthadesmia, como modelo de estudio para el análisis biogeográfico, dado que posee el mayor número de especies (33 especies), y a su vez, abarca gran parte de la distribución del género en América del Sur. Las especies de la serie Microphyllae han sido descritas como xerófitas que habitan el sur de Perú y Bolivia; Chile y Argentina poseen el mayor número de especies (22 especies nativas para cada país, de las cuales son endémicas el 41 y el $37 \%$, respectivamente). Si bien son esencialmente andinas, en Chile y Argentina existen especies que habitan a nivel del mar (Tabla 1).

TABLA 1

Distribución geográfica y altitudinal de las especies de la serie Microphyllae; ar = Argentina, bo $=$ Bolivia, $\mathrm{ch}=$ Chile, pe $=$ Perú

Geographic and altitudinal distributions of Microphyllae species; ar $=$ Argentina, bo $=$ Bolivia, ch $=$ Chile, pe $=$ Perú

\begin{tabular}{|c|c|c|}
\hline Especie & Rango altitudinal (m) & País \\
\hline Adesmia acuta Burkart & $1.500-3.000$ & ar \\
\hline A. adrianii Correa & $1.000-1.500$ & ar \\
\hline A. aegiceras Philippi & $2.000-4.000$ & ar-ch \\
\hline A. arenicola (Fries) Burkart & $3.000-3.500$ & ar \\
\hline A. augusti Macbride & 2.900 & pe \\
\hline A. confusa Ulibarri & 800 & $\mathrm{ch}$ \\
\hline A. dessaueri (Reiche) Ulibarri & Sin información & $\mathrm{ch}$ \\
\hline A. erinacea Philippi & $2.500-4.000$ & ar-ch \\
\hline A. friesii Burkart ex Ulibarri & $2.000-3.000$ & ar \\
\hline A. gracilis Meyen ex Vogel & $0-3.000$ & ar-ch \\
\hline A. hirsuta Philippi & Sin información & $\mathrm{ch}$ \\
\hline A. horrida Gillies ex Hooker et Arnott & $2.500-4.000$ & ar bo ch \\
\hline A. hystrix Philippi & $2.400-3.800$ & $\mathrm{ch}$ \\
\hline A. melanocaulos Philippi & Sin información & $\mathrm{ch}$ \\
\hline A. melanthes Philippi & $2.000-4.000$ & ch pe \\
\hline A. mendozana Ulibarri & $1.500-2.400$ & ar \\
\hline A. microphylla Hooker et Arnott & $400-1.000$ & ch \\
\hline A. miraflorensis Remy & $3.000-4.000$ & ar-bo-pe \\
\hline A. obcordata Clos & $0-2.500$ & ar-ch \\
\hline A. obovata Clos & $500-2.000$ & ar-ch \\
\hline A. pauciflora Vogel & $1.600-1.700$ & $\mathrm{ch}$ \\
\hline A. pentaphylla Philippi & $2.000-3.000$ & ar-ch \\
\hline A. pinifolia Gill. ap. Hooker et Arnott & $1.500-4.000$ & ar-ch \\
\hline A. polyphylla Philippi & 4.000 & $\mathrm{ch}$ \\
\hline A. pumahuasiana Ulibarri & $3.000-4.000$ & ar-bo \\
\hline A. pungens Clos & $100-180$ & $\mathrm{ch}$ \\
\hline A. renjifoana (Reiche) Ulibarri & $1.500-3.000$ & ar-ch \\
\hline A. sanjuanensis Burkart & $2.000-3.000$ & ar \\
\hline A. schneideri Philippi & $0-2.500$ & ar-ch \\
\hline A. spinosissima Meyen & $2.500-5.000$ & ar-bo-ch-pe \\
\hline A. trijuga Gill. ex Hooker et Arnott & $1.000-3.000$ & ar \\
\hline A. uspallatensis Gill. Ex Hooker et Arnott & $1.500-3.000$ & ar \\
\hline A. volckmannii Philippi & $0-2.500$ & ar-ch \\
\hline
\end{tabular}




\section{Métodos}

La panbiogeografía involucra el establecimiento de una relación general entre la distribución de los organismos y la historia de la tierra. En este tipo de análisis se pone énfasis en la importancia de la dimensión geográfica o espacial de la diversidad de la vida, destacándose el papel de las distribuciones geográficas como objetos de análisis directo (Morrone \& Crisci 1995, Craw et al. 1999). Entre las diferentes metodologías para realizar un análisis panbiogeográfico se encuentran la reconstrucción manual (Croizat 1958, 1964), las matrices de conectividad e incidencia (Page 1987), la compatibilidad de trazos (Craw 1988, 1989) y el análisis de parsimonia de endemismos (Craw et al. 1999, Morrone \& Márquez 2001). Para la construcción del modelo biogeográfico en la serie Microphyllae empleamos la reconstrucción manual y el análisis de parsimonia de endemismos (PAE).

En los análisis realizados, la distribución de las especies fue tomada de Ulibarri (1987). Con el programa ArcView 3.2 se confeccionaron distintas capas de información para cada una de las especies, a partir de las cuales se determinaron los trazos individuales y generalizados. En la actualidad, diversos criterios han sido propuestos para la construcción de trazos individuales (ver discusión de Henderson 1989 y Craw et al. 1999). En este trabajo, el procedimiento utilizado para obtener los trazos individuales fue el de árbol de tendido mínimo ("minimum spanning tree"), uniendo las localidades geográficas a través de la línea de menor distancia calculada en los mapas antes mencionados. La unión del vecino geográfico más cercano es un procedimiento empleado cuando no existe otro tipo de información, como por ejemplo la proveniente de la filogenia, para construir el árbol de tendido mínimo (Craw et al. 1999). Si bien una vez que se obtienen los trazos es posible orientarlos, empleando los criterios de línea de base, centro de masa e información filogenética, solo trabajamos con trazos no orientados, dado que no existe información suficiente para aplicar estos criterios.

Cuando varios trazos individuales coinciden se considera la existencia de un trazo generalizado. Este proporciona una hipótesis de homología biogeográfica primaria, que permite sostener la existencia de una biota ancestral ampliamente distribuida y fragmentada por algún evento vicariante. Los trazos generalizados planteados en este artículo consideran, además de la coincidencia total, la coincidencia parcial de los trazos individuales para establecer un trazo generalizado; esta alternativa permite que un trazo individual pueda pertenecer a más de un trazo generalizado, esto sería análogo a la metodología utilizada por diversos autores para encontrar relaciones no jerárquicas entre áreas en un análisis de parsimonia de endemismos (Luna et al. 1999, García Barros et al. 2002, Morrone 2004a). Al "desconectar" las sinapomorfias que sustentan los clados, y reanalizar la matriz, es posible incorporar la posibilidad de que las áreas se relacionen con distintas agrupaciones (clados), flexibilizando la jerarquía establecida por el método.

Cuando dos o más trazos generalizados se interceptan se define un nodo. Los nodos implican un origen biótico múltiple y en ocasiones se interpretan como zonas de convergencia tectónica, donde fragmentos de dos o más regiones bióticas y geológicas entran en contacto y se combinan (Zunino \& Zullini 2003).

En la actualidad, el PAE es considerado como uno de los métodos para realizar análisis panbiogeográficos, donde los clados obtenidos son considerados como trazos generalizados (Craw et al. 1999, Morrone \& Márquez 2001). Para este análisis, el área total de distribución de las especies fue dividida en unidades de tamaño diferente (Posadas \& Miranda-Esquivel 1999), en primer lugar el área fue dividida en cuadrículas de $1^{\circ} \mathrm{x} 1^{\circ}$ (Anexo 1A), luego en franjas latitudinales de $1^{\circ}$ (Anexo 1B) y por último en franjas longitudinales de $1^{\circ}$ (Anexo 1C). Siguiendo a Morrone (2004a), en los tres casos las matrices resultantes de áreas $\mathrm{x}$ trazos individuales fueron codificadas como presencia (1) o ausencia (0) del trazo individual de cada especie en las distintas cuadrículas. Este método agrupa áreas o localidades, optimizando la concordancia entre los patrones de distribución de muchos taxones diferentes, utilizando para ello los taxones compartidos entre áreas de acuerdo con la solución más parsimoniosa (Morrone 1994b, Posadas 1996). Las matrices de áreas $\mathrm{x}$ trazos individuales 
fueron analizadas con los programas NONA 2.0 (Goloboff 1993) y WinClada (Nixon 1999). La estrategia de búsqueda de los cladogramas más parsimoniosos se realizó mediante búsquedas heurísticas con permutación de ramas tipo TBR y reconexión múltiple (Múltiple TBR + TBR), permitiéndole al programa retener un máximo de 10.000 árboles, con 100 réplicas de secuencia de adición al azar y 100 árboles iniciales en cada repetición (árboles de Wagner). Se consideró solo aquellos caracteres informativos.

Para optimizar los cladogramas resultantes se utilizó la opción ACTRAN de WinClada, que en el caso de existir más de una alternativa igualmente posible para determinar la secuencia de transformación en los caracteres (en este caso la presencia de las especies), favorece los cambios en las ramas basales del cladograma. Las homoplasias fueron interpretadas como reversiones, que en un contexto biogeográfico equivaldrían a eventos de extinción. Se prefirió esta interpretación debido a que las explicaciones alternativas de la homoplasia (e.g., convergencia y paralelismo), corresponderían a eventos de dispersión. La dispersión se plantea como un escenario poco probable para el género, debido a características como son: frutos dehiscentes, distribuciones restringidas en muchas de las especies y, hasta la fecha, ningún dispersor conocido.

En todos los análisis se obtuvo el cladograma de consenso estricto, que fue utilizado para evaluar las áreas agrupadas y las posibles causas históricas que las expliquen (Morrone 1994b).

\section{RESULTADOS}

\section{Patrones de distribución y trazos individuales}

En la Fig. 1A-G se representa la distribución geográfica y los trazos de individuales de las especies de la serie Microphyllae. Para el análisis panbiogeográfico no fueron consideradas las especies A. acuta, A. adrianii, A. arenicola, A. augusti, A. dessaueri, A. friesii, A. hirsuta, A. melanocaulos, A. pauciflora, A. sanjuanensis y A. trijuga, debido a que no se disponía información de al menos tres localidades geográficas de su distribución, que constituiría el número mínimo de localidades para establecer un trazo individual informativo (Grehan 2001).

Con excepción de las especies A. pungens y A. microphylla (Fig. 1C y 1F), todas las especies presentan una distribución asociada con la Cordillera de los Andes. Las especies $A$. obcordata, A. volckmannii, A. horrida y A. schneideri presentan las distribuciones más amplias. Longitudinalmente, A. spinosissima y A. miraflorensis se destacan por su amplitud mayor. El resto de las especies presenta una distribución restringida latitudinal y longitudinalmente (Fig. 1A-G).

\section{Trazos generalizados}

Se hallaron cinco trazos generalizados (Fig. 2); en la Tabla 2 se presenta la composición de cada uno de ellos. De acuerdo con los patrones encontrados, se determinó la presencia de dos nodos (Fig. 2, Tabla 2). El nodo de la zona norte, que contiene los vértices de los trazos 1b, 2c y 3a; y el nodo de la zona central, que contiene los vértices de los trazos 3c, 4a y 5 . Las especies A. gracilis, A. mendozana, A. pentaphylla y $A$. renjifoana presentaban una distribución muy restringida, por lo cual no fue posible asociarlas con ninguno de los trazos propuestos, sin embargo, todas se encuentran asociadas con el nodo II.

\section{Análisis de parsimonia de endemismos}

En el análisis considerando las cuadrículas de $1^{\circ} \times 1^{\circ}$ se obtuvieron 10.000 cladogramas de máxima parsimonia de los 10.000 permitidos en el análisis, cuyos estadísticos son: largo (L): 64, índice de consistencia (CI): 0.34 e índice de retención (RI): 0.83 (Fig. 3). El análisis a esta escala produjo muchos cladogramas con poca resolución y con pocas especies exclusivas (sinapomorfías) que sustenten las agrupaciones. El cladograma de consenso posee una politomía basal, donde se destaca la agrupación de las cuadrículas 720-721- 752 en la zona norte (Fig. $3)$, localizados aproximadamente a los $20^{\circ}$ de latitud sur, y cuatro grupos a la altura de Chile central, entre $\operatorname{los} 30^{\circ}$ y $35^{\circ} \mathrm{S}$, correspondientes a las cuadrículas 930-953, 954-977-999, 1.0391.020-1.040-1.060, y 975-1.018-976-998 -1.019 (Fig. 3). Ninguna de las agrupaciones presentó más de una sinapomorfía. 

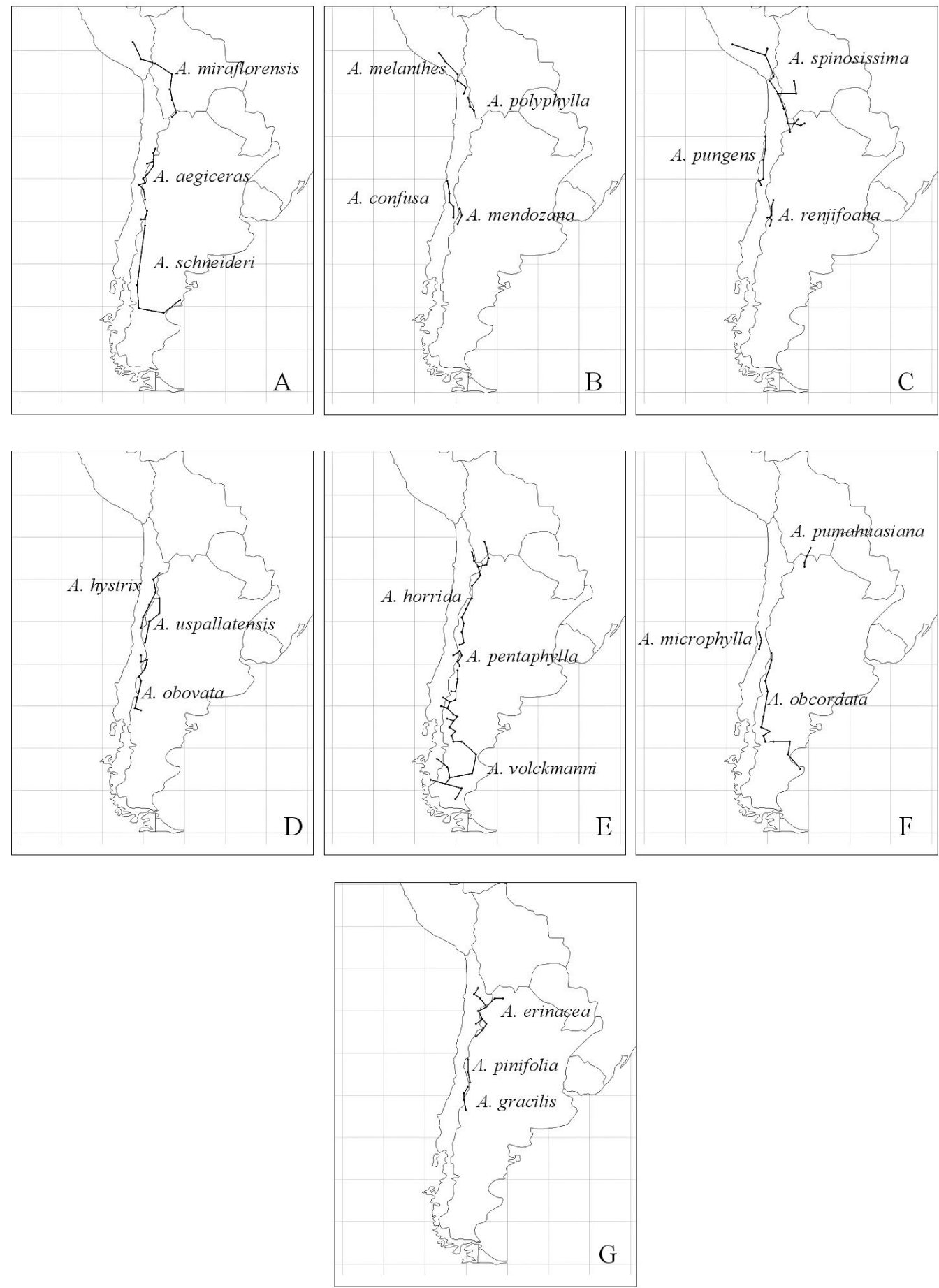

Fig. 1: Trazos individuales de las especies de la serie Microphyllae. A: A. aegiceras, A. miraflorensis, A. schneideri. B: A. confusa, A. melanthes, A. mendozana, A. poliphylla. C: A. pungens, A. renjifoana, A.spinosissima. D: A. hystrix, A. obovata, A. uspallatensis. E: A. horrida, A. pentaphylla, A. volckmanni. F: A. microphylla, A. obcordata, A. pumahuasiana. G: A. erinacea, A. gracilis, A. pinifolia.

Individual tracks of the species of the series Microphyllae. A: A. aegiceras, A. miraflorensis, A. schneideri. B: A. confusa, A. melanthes, A. mendozana, A. poliphylla. C: A. pungens, A. renjifoana, A.spinosissima. D: A. hystrix, A. obovata, A. uspallatensis. E: A. horrida, A. pentaphylla, A. volckmanni. F: A. microphylla, A. obcordata, A. pumahuasiana. G: A. erinacea, A. gracilis, A. pinifolia. 


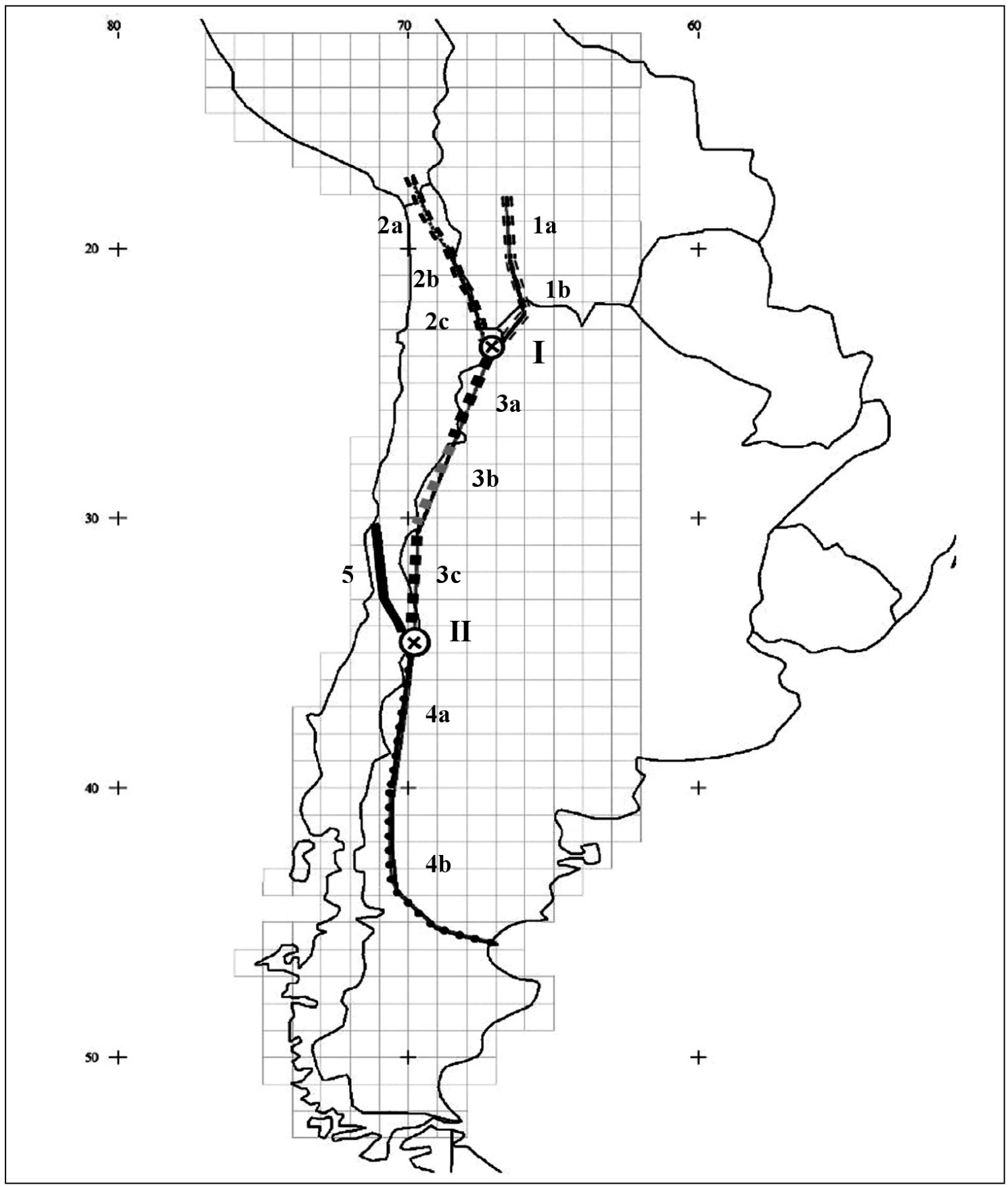

Fig. 2: Trazos generalizados y nodos propuestos para la serie Microphyllae. El círculo con una $\mathrm{x}$ indica la presencia de un nodo.

Generalized tracks and nodes proposed for the series Microphyllae. The circle with an $\mathrm{x}$ indicates the presence of a node. 
TABLA 2

Composición de los elementos encontrados en el análisis de trazos

Composition of the elements found in track analysis

\begin{tabular}{|c|c|}
\hline Trazo & Especie \\
\hline \multirow[t]{2}{*}{1} & A. miraflorensis, A. spinosissima \\
\hline & A. miraflorensis, A. horrida \\
\hline \multirow[t]{3}{*}{2} & A. spinosissima, A. melanthes \\
\hline & A. spinosissima, A. polyphylla \\
\hline & A. spinosissima, A. horrida \\
\hline \multirow[t]{3}{*}{3} & A. horrida, A. hystrix, A. erinacea \\
\hline & A. horrida, A. hystrix, A. aegiceras, A. uspallatensis \\
\hline & A. horrida, A. aegiceras, A. uspallatensis, A. pinifolia \\
\hline \multirow[t]{2}{*}{4} & A. obcordata, A. schneideri, A. volckmannii, A. obovata \\
\hline & A. obcordata, A. schneideri, A. volckmannii \\
\hline 5 & A. confusa, A. microphylla, A. pungens \\
\hline Nodo I & Trazos $1 b, 2 c, 3 a$ \\
\hline Nodo II & Trazos 3c, 4a y 5, Además de las especies: A. gracilis, A. mendozana, A.pentaphylla y A. renjifoana \\
\hline
\end{tabular}

Al considerar las franjas latitudinales, el PAE produjo cuatro cladogramas igualmente parsimoniosos (L: 35, CI: 0.62, RI: 0.90). El análisis agrupó las cuadrículas en dos clados (Fig. 4). De acuerdo con esto, el área de distribución de las especies de la serie Microphyllae puede ser dividida en una zona norte y una zona sur, el límite entre ambos grupos se encuentra en Chile central, aproximadamente a los $33^{\circ} \mathrm{S}$ (Fig. 4). En esta agrupación, las especies $A$. miraflorensis en la zona norte y A. volckmannii en la zona sur, son las únicas que aparecen como sinapomorfias que respaldan la división norte-sur. Sin embargo, debido a que estas especies no se encuentran presentes en todos los terminales del clado, su ausencia puede ser interpretada como eventos de extinción (reversiones). El cladograma de consenso muestra patrones anidados tanto para las cuadrículas de la zona norte como para las de la zona sur. Integrando ambos resultados del análisis de parsimonia, la agrupación de las cuadrículas 975-976-9981.018-1.019 del análisis de $1^{\circ} \times 1^{\circ}$ mezcla cuadrículas tanto de la zona norte como de la zona sur obtenidas en el segundo análisis. El análisis con las franjas longitudinales de $1^{\circ}$ produjo cinco cladogramas igualmente parsimoniosos (L:32, CI: 0,62 y RI: 0,75); en el cladograma de consenso se refleja la mayor concentración de especies en las cuadrículas que involucran la porción de la Cordillera de los Andes ubicadas en la zona de Chile Central, estando sustentada por un número mayor de sinapomorfias y apareciendo como una dicotomía terminal en el cladograma (Fig. 5).

$\mathrm{Al}$ considerar en conjunto los resultados del análisis de trazos y del PAE es posible entender de manera más integral la naturaleza de los patrones observados. Por ejemplo, la zona norte de la distribución de la serie contiene los trazos 1, 2, 3 y 5; y la zona sur al trazo 4. El límite entre ambas se encuentra dado por el nodo II. Los trazos 1 y 2 se encuentran en la vertiente oriental de la Cordillera de los Andes, mientras que el trazo 5 es el único establecido para la vertiente occidental de la cordillera y el trazo 3 se dispone en plena cordillera. Los trazos individuales de las especies que definen al trazo generalizado 5 se asocian con la depresión intermedia y la cordillera de la costa de Chile, y solo la porción más meridional del trazo se encontraría asociada con la cordillera de los Andes. 


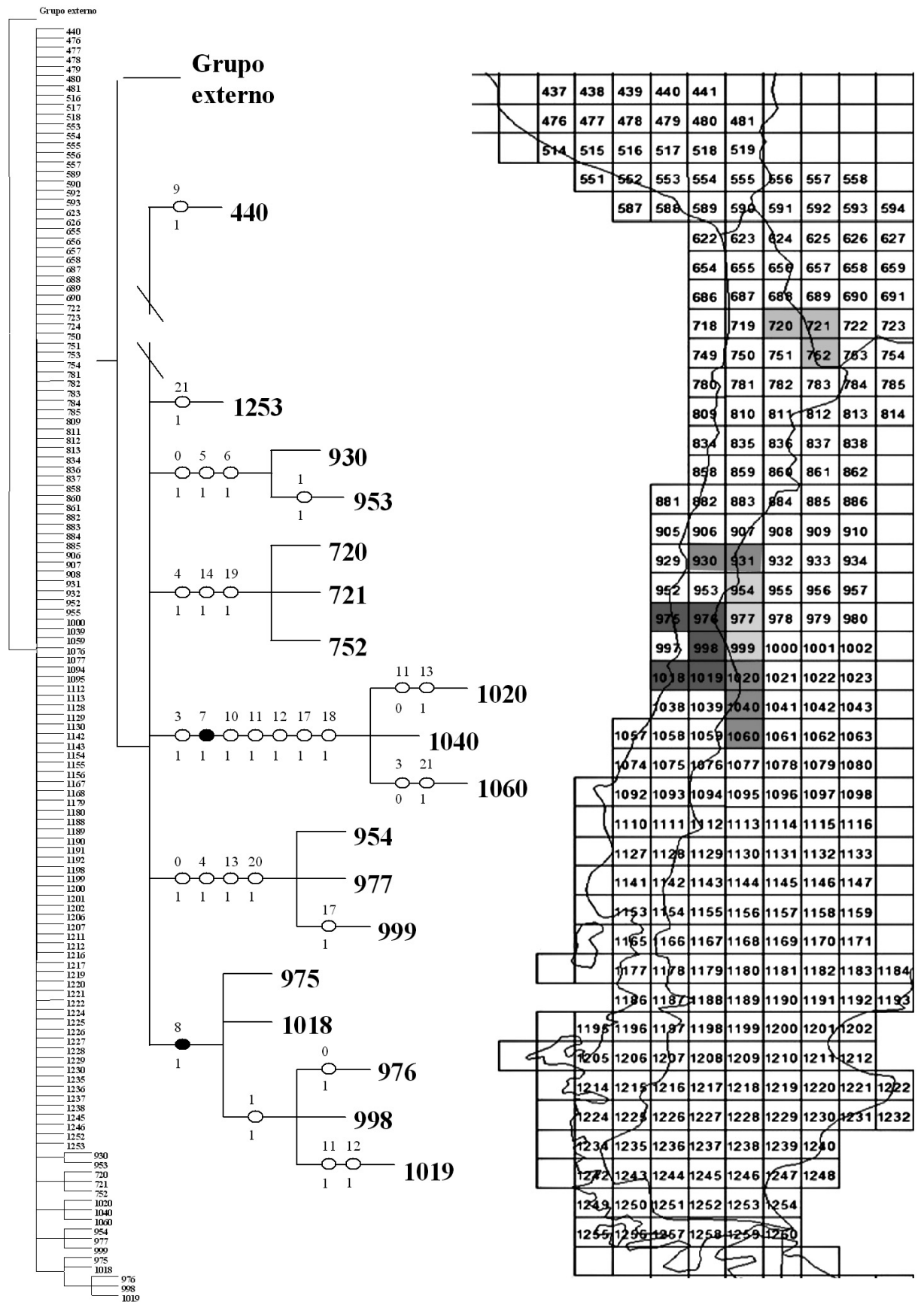

Fig. 3: Cladograma de consenso de los 10.000 cladogramas igualmente parsimoniosos (L: 64, CI: 0,4; RI: 0,83) obtenidos del PAE con una cuadrícula de $1^{\circ} \times 1^{\circ}$. Codificación de caracteres: $A$. aegiceras (0), A. confusa (1), A. erinacea (2), A. gracilis (3), A. horrida (4), A. hystrix (5), A. melanthes (6), A. mendozana (7), A. microphylla (8), A. miraflorensis (9), A. obcordata (10), A. obovata (11), A. pentaphylla (12), A. pinifolia (13), A. poliphylla (14), A. pumahuasiana (15), A. pungens (16), A. renjifoana (17), A. schneideri (18), A.spinosissima (19), A. uspallatensis (20), A. volckmanni (21).

Consensus cladogram of the 10,000 cladograms obtained from the PAE with $1^{\circ} \mathrm{x} 1^{\circ}$ quadrats (L: 64, CI: $0.34, \mathrm{RI}: 0.83$ ) Codification of characters: A. aegiceras (0), A. confusa (1), A. erinacea (2), A. gracilis (3), A. horrida (4), A. hystrix (5), A. melanthes (6), A. mendozana (7), A. microphylla (8), A. miraflorensis (9), A. obcordata (10), A. obovata (11), A. pentaphylla (12), A. pinifolia (13), A. poliphylla (14), A. pumahuasiana (15), A. pungens (16), A. renjifoana (17), A. schneideri (18), A. spinosissima (19), A. uspallatensis (20), A. volckmanni (21). 


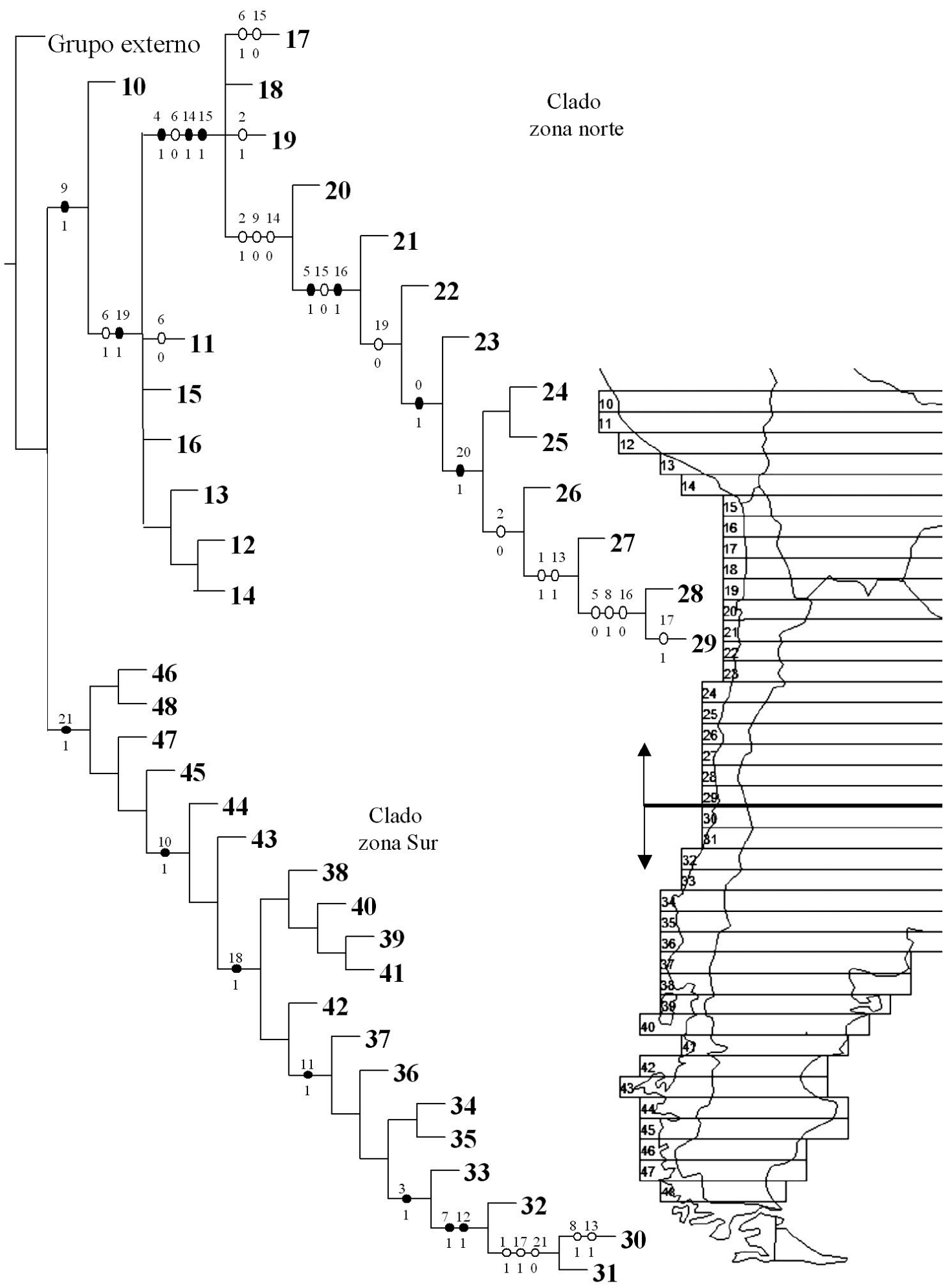

Fig. 4: Cladograma de consenso de los cuatro cladogramas igualmente parsimoniosos (L: 35, CI: 0,62, RI: 0,90) del PAE con franjas latitudinales de $1^{\circ}$. Codificación de caracteres es la misma que la de la Fig. 3.

Consensus cladogram of the four cladograms obtained with PAE with latitudinal belts of $1^{\circ}$ (L: 35 , CI: $0.62, \mathrm{RI}$ : 0.90 ). Codification of characters is the same that Fig. 3. 


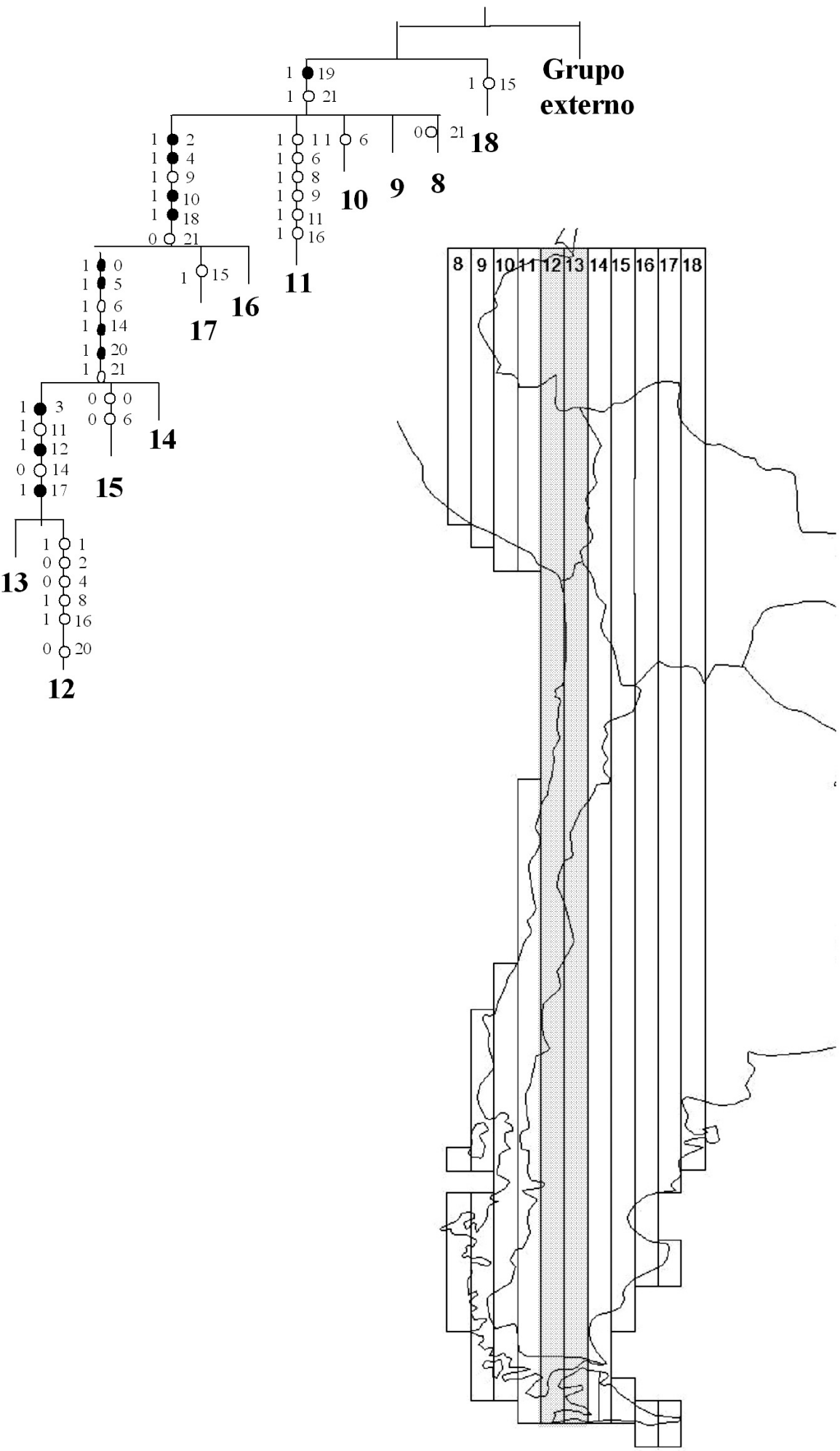

Fig. 5: Cladograma de consenso de los cinco cladogramas igualmente parsimoniosos (L:32, CI: 62 y RI: 75) obtenidos en el PAE con franjas longitudinales de $1^{\circ}$. Codificación de caracteres es la misma que la de la Fig. 3.

Consensus cladogram of the five cladograms obtained with PAE with longitudinal belts of $1^{\circ}$ (L: L:32, CI: 62 y RI: 75). Codification of characters is the same one that Fig. 3. 


\section{DISCUSIÓN}

Los antecedentes disponibles de la historia biogeográfica del género Adesmia son escasos, contándose en la actualidad solo con algunas aproximaciones que mencionan el tema de manera superficial o anecdótica. El modelo que se postula a continuación se plantea como una hipótesis inicial, en otras palabras, la primera fase de análisis biogeográfico para el género.

Los patrones coincidentes de los trazos individuales de la mayor parte de las especies analizadas permiten plantear que los patrones de distribución actual de las especies habrían estado determinados, principalmente, por eventos vicariantes. A partir de los resultados obtenidos se postula que el desarrollo del macizo cordillerano estaría involucrado en la diferenciación reciente de las especies de la serie Microphyllae, concordando parcialmente con lo propuesto por Burkart (1967). El alzamiento de los Andes habría fragmentado la distribución de las especies (vicarianza), ocasionando una diferenciación de grupos posterior (posiblemente asociados a biotas diferentes). Esto explicaría el alto número de especies con distribución restringida y la homogeneidad morfológica entre ellas. Burkart (1967) describió la existencia de dos centros de polimorfismos para Adesmia: uno en la Cordillera de los Andes frente a Chile central, y otro en las regiones áridas de Cuyo, Catamarca, Salta, hacia el este de la Cordillera de los Andes. El primero de los centros descritos coincidiría con el nodo II.

El desarrollo de la Cordillera de los Andes y regiones adyacentes ha sido un proceso paulatino que ha abarcado decenas de millones de años, existiendo diferencias marcadas en el tipo y antigüedad de sus elevaciones (James 1973, Taylor 1991, 1995). Adicionalmente, los cambios climáticos, como las glaciaciones del Pleistoceno (Simpson \& Todzia 1990), podrían haber favorecido la ocurrencia de eventos locales de vicarianza y diversificación periférica, esto permitiría explicar que algunas especies, dependiendo de sus capacidades intrínsecas de adaptación y de cuan amplio era el rango distribución al momento del evento de fragmentación, pudiesen formar parte de más de un trazo generalizado, como es el caso de $A$. miraflorensis, A. horrida y A. spinosissima, entre otras.
Los patrones, no jerárquicos, presentados por estas especies, a su vez pueden ser causales de la escasa resolución encontrada en el PAE, como son la presencia de politomías, pocas sinapomorfias, bajos índices de consistencia y de retención, debido a que estas especies tienen igual probabilidad de asociación con una u otra agrupación (igual probabilidad de pertenecer a uno u otro trazo generalizado). No obstante, en términos generales, los resultados obtenidos son concordantes con el modelo planteado, evidenciándose en las agrupaciones el nodo II, en el análisis de $1^{\circ} \mathrm{x} 1^{\circ}$, agrupación de las cuadrículas que involucran el macizo cordillerano en el análisis de franjas longitudinales.

En particular, la zona de la Puna ha sido catalogada como una zona compleja, debido a las relaciones de elementos bióticos distintos. Esta área formaría parte de la Zona de Transición Sudamericana, donde entran en contacto elementos bióticos neotropicales y andinos (Morrone 2004b). En este tipo de áreas se espera la presencia de una alta concentración de nodos panbiogeográficos, debido al intercambio de especies procedentes de biotas de distinto origen. En este sentido, es importante mencionar que el nodo encontrado en la zona norte sería coincidente con el nodo de la Puna (Katinas et al. 1999), donde se interceptan los trazos generalizados andino y tropical. Cabe destacar que los trazos generalizados 3 y 4 también se pueden relacionar con los trazos generalizados andinos endémicos establecidos en el mismo trabajo, los cuales comprenderían las subregiones Chilena Central, Patagónica y Subantártica para el trazo 3; y Subantártica y Patagónica para el trazo 4.

En cuanto a los procesos, a lo largo de la historia de la biogeografía han existido importantes debates para establecer cuál es el más importante. En la actualidad, la biogeografía posee varias metodologías que pretenden explicar y/o identificar procesos tales como dispersión y vicarianza, fenómenos no excluyentes que ocurren en la naturaleza y que constituyen explicaciones alternativas para las distribuciones disjuntas (Nelson \& Platnick 1980, 1981). El análisis realizado en este estudio indica que la vicarianza sería el proceso que determinó los actuales patrones de distribución en la serie, donde la separación no habría ocurrido en sentido longitudinal, es decir, entre ambas vertientes de la cordillera, si 
no más bien en sentido latitudinal, como lo indica la secuencia de trazos generalizados dispuestos latitudinalmente.

La relación estrecha entre la sistemática y la biogeografía de los organismos (Zunino \& Zullini 2003) permite una retroalimentación entre ambas disciplinas, a partir de la cual las distintas hipótesis tanto sistemáticas como biogeográficas, pueden ser corroboradas o refutadas. No obstante, las hipótesis de relaciones históricas entre distintas entidades (taxa, áreas, biotas) resultan independientes de la filogenia de sus ocupantes, así como de las reconstrucciones paleogeográficas y paleoclimáticas. Esto permite aplicar el principio de elucidación recíproca a las diferentes hipótesis que atañen al mismo fenómeno, pero que son totalmente autónomas y que al tener el mismo nivel de legitimidad científica, permite su comparación en forma paritaria (Zunino 2000, Morrone 2001c). En el caso de Adesmia, Burkart (1967) estableció su clasificación infragenérica, donde las especies utilizadas en este estudio fueron tratadas como dos series independientes: Microphyllae y Arboreae Burkart. Sin embargo, dada la complejidad del grupo, este autor reconoció como poco resueltas las relaciones de las especies entre las series y al interior de las mismas. Posteriormente, Ulibarri (1987) realizó la revisión de estas dos series, y estableció su sinonimia, junto con la de numerosas especies. Los resultados obtenidos en este trabajo indicarían que las especies estudiadas comparten una historia, y que en ausencia de más antecedentes, se trataría de un grupo natural, brindando apoyo a la hipótesis de Ulibarri (1987).

\section{AGRADECIMIENTOS}

Al Departamento de Biología Evolutiva de la Facultad de Ciencias de la Universidad Nacional Autónoma de México, por las facilidades otorgadas a la autora principal para la realización de la estadía en sus dependencias. Se agradece además el gran apoyo de Noé Velázquez y Yaayé Arellanes, y los comentarios, revisión y sugerencias de Alicia Marticorena, y la mejora en las imágenes de Nicole Fierro Nova. Investigación financiada por Proyecto Comisión Nacional de
Investigación Científica y Tecnológica de Chile (CONICYT) AT-4040202, y Proyecto Programa de Mejoramiento de la Calidad y la Equidad de la Educación Superior (MECESUP) UCO 0214. Esta investigación forma parte de las actividades del Núcleo Milenio de Estudios Avanzados en Ecología e Investigaciones en Biodiversidad P02-054 F ICM.

\section{LITERATURA CITADA}

BURKART A (1967) Contribución al estudio del género Adesmia DC. VII. Sinopsis del género sudamericano de leguminosas Adesmia DC. Darwiniana (Argentina) 14: 463-568.

BURKART, A \& M N CORREA (1984) Adesmia DC. En M N Correa (Ed.). Flora Patagónica 8 (4b): 92-161. Colección Científica del Instituto Nacional de Tecnología Agropecuaria (INTA), Buenos Aires, Argentina.

CRAW RC (1988) Continuing the synthesis between panbiogeography, phylogenetic systematics and geology as illustrated by empirical studies on the biogeography of New Zealand and the Chatham Islands. Systematic Zoology 37: 291-310.

CRAW RC (1989) New Zealand biogeography: A panbiogeographic approach. New Zealand Journal of Zoology 16: 527-547.

CRAW RC, JR GREHAN \& M J HEADS (1999) Panbiogeography: tracking the history of life. Oxford University Press, New York, USA. 229 pp.

CRISCI JV, MM CIGLIANO, JJ MORRONE \& S ROIGJUNENT (1991) Historical biogeography of southern South America. Systematic Zoology 40: 152-171.

CROIZAT L (ed) (1958) Panbiogeography. Volumes 1 \& 2. Caracas, Venezuela. $1.731 \mathrm{pp}$.

CROIZAT L (ed) (1964) Space, time, form: the biological synthesis. Caracas, Venezuela. $881 \mathrm{pp}$.

DAVYT M \& P IZAGUIRRE (1996) Sinopsis de las especies y variedades del género Adesmia DC (Fabaceae- Adesmieae) en el Uruguay. Parodiana (Argentina) 9: 89-114.

EZCURRA CE, A RUGGIERO \& JV CRISCI (1997) Phylogeny of Chuquiraga sect. Acanthophyllae (Asteraceae-Barnadesioideaee), and the evolution of its leaf morphology in relation to climate. Systematic Botany 22: 151-163.

GARCÍA BARROS E, P GURREA, MJ LUCIÁÑEZ, JM CANO, ML MUNGUIRA, JC MORENO, H SAINZ, MJ SANZA \& JC SIMÓN (2002) Parsimony analysis of endemicity and its application to animal and plant geographical distributions in the IberoBalearic region (western Mediterranean). Journal of Biogeography 29: 109-124.

GOLOBOFF P (1993) NONA ver 2.0. Fundación e Instituto Miguel Lillo, Tucumán, Argentina. http:// www.cladistics.com

GREHAN JR (2001) Panbiogeografía y la geografía de la vida. En: Llorente J \& J Morrone (eds) Introducción a la biogeografía en Latinoamérica: teoría, conceptos, métodos y aplicaciones: 181-195. Las Prensas de Ciencias, Facultad de Ciencias, Universidad Nacional Autónoma de México, México Distrito Federal, México. 
HENDERSON IM (1989) Quantitative panbiogeography: An investigation into concepts and methods. New Zealand Journal of Zoology 16: 495-510.

HERNÁNDEZ J, T WALSHBURGER, R ORTIZ \& A HURTADO (1992) Origen y distribución de la biota sudamericana y colombiana. En: Halffter G (compilador) La diversidad biológica de Iberoamérica: 55-104. Acta Zoológica Mexicana, Xalapa, México.

HINOJOSA LF \& C VILLAGRÁN (1997) Historia de los bosques del sur de Sudamérica, I: antecedentes paleobotánicos, geológicos y climáticos del Terciario del cono sur de América. Revista Chilena de Historia Natural 70: 225- 239.

JAMES D (1973) The evolution of the Andes. Scientific American 229: 60-69.

KATINAS L, JJ MORRONE \& JV CRISCI (1999) Track analysis reveals the composite nature of the Andean biota. Australian Journal of Botany 47: 111-130.

LUNA I, O ALCÁNTARA, D ESPINOSA \& JJ MORRONE (1999) Historical relationships of the Mexican cloud forests: a preliminary vicariance model applying Parsimony Analysis of Endemicity to vascular plant taxa. Journal of Biogeography 26: 1299-1305.

MIOTTO ST (1993) Quatro spécies novas de Adesmia DC. (Leguminosae-Faboideae) do Sul do Brasil. Bradea 7: $248-258$

MORRONE JJ (1994a) Systematics, cladistics and biogeography of the Andean weevil genera Macrostyphlus, Adioristidius, Puranius, and Amathynetoides, new genus (Coleoptera: Curculionidae). American Museum Novitates 3104: 1-63.

MORRONE JJ (1994b) On the identification of areas of endemism. Systematic Biology 43: 438-441.

MORRONE JJ (1996) The biogeographical Andean subregion: a proposal exemplified by Arthropod taxa (Aracnida, Crustacea, and Hexapoda). Neotropica (Argentina) 42: 103-114.

MORRONE JJ (2001a) Biogeografía de América Latina y el Caribe. Manuales \& Tesis Volumen 3. Sociedad Entomológica Aragonesa (SEA). Programa Iberoamericano de Ciencia y Tecnología para el Desarrollo (CYTED). Oficina Regional de Ciencia y Tecnología de United Nations Educational, Scientific and Cultural Organization para América Latina y el Caribe (ORCYT-UNESCO), Sociedad Entomológica, Aragonesa (SEA), Zaragoza, España. 148 pp.

MORRONE JJ (2001b) Homology, biogeography and areas of endemism. Diversity and Distributions 7: 297-300.

MORRONE JJ (2001c) Sistemática, biogeografía, evolución: los patrones de la biodiversidad en tiempo-espacio. Las Prensas de Ciencias, Universidad Nacional Autónoma de México, México Distrito Federal, México. 124 pp.

MORRONE JJ (2002) Presentación sintética de un nuevo esquema biogeográfico de América Latina y el Caribe. En: Costa C, S Vanin, J Lobo \& A Melic (eds) Proyecto de Red Iberoamérica de Biogeografía y Entomología Sistemática PrIBES 2002. m3m-Monografías Tercer Milenio, Sociedad Entomológica Aragonesa (SEA), Zaragoza, España. $326 \mathrm{pp}$.

MORRONE J J (2004b) La Zona de Transición Sudamericana: caracterización y relevancia evolutiva. Acta Entomológica Chilena 28: 41-50.

MORRONE JJ (2004a) Panbiogeografía, componentes bióticos y zonas de transición. Revista Brasileira de Entomologia 48: 149-192.
MORRONE JJ \& JV CRISCI (1995) Historical biogeography: introduction to methods. Annual Review of Ecology and Systematics 26: 373-401.

MORRONE JJ \& J MÁRQUEZ (2001) Halffter's Mexican Transition Zone, beetle generalised tracks, and geographical homology. Journal of Biogeography 28: 635-650.

NELSON G \& NI PLATNICK (1980) A vicariance approach to historical biogeography. Bioscience 30 : 339-343.

NELSON G \& NI PLATNICK (1981) Systematics and Biogeography: Cladistics and Vicariance. Columbia University Press, New York, New York, USA. 567 pp.

NIXON K C (1999) Winclada (BETA) ver. 0.9.9 published by the author, Ithaca, New York, USA. http:// www.cladistics.com.

NORES M \& MM CERRANA (1990) Biogeography of forest relics in the mountains of northwestern Argentina. Revista Chilena de Historia Natural 63: 37-46.

PAGE R DM (1987) Graphs and generalized tracks: quantifying Croizat's panbiogeography. Systematic Zoology 36: 1-17.

POSADAS P (1996) Distributional patterns of vascular plants in Tierra del Fuego: a study applying parsimony analysis of endemicity (PAE). Biogeographica 72: 161-177

POSADAS P \& D MIRANDA- ESQUIVEL (1999) El PAE (Parsimony Analysis of Endemicity) como una herramienta en la evaluación de la biodiversidad. Revista Chilena de Historia Natural 72: 539-546.

SIMPSON B (1983) An historical phytogeography of the high Andean flora. Revista Chilena de Historia Natural 56: 109-122.

SIMPSON S \& C TODZIA (1990) Patterns and processes in the development of the high Andean flora. American Journal of Botany 77: 1419-1432.

TAYLOR D (1991) Palaeobiogeographic relationships of Andean angiosperms of Cretaceous to Pliocene age. Palaeogeography, Palaeoclimatology \& Palaeoecology 88: 69-84.

TAYLOR D (1995) Cretaceous to Tertiary geologic and angiosperm paleobiogeographic history of the Andes. En: Churchill S, Balslev H, Forero E \& J Luteyn (Eds) Biodiversity and conservation of Neotropical montane forests: 3-9 pp. The New York Botanical Garden, New York, New York, USA.

ULIBARRI EA (1980) Notas sobre Adesmia DC. I (Leguminosae- Papilionoideae). Darwiniana (Argentina) 22: 493-498.

ULIBARRI EA (1982) Notas sobre Adesmia DC. II (Leguminosae- Papilionoideae). Darwiniana (Argentina) 24: 267-281.

ULIBARRI E A (1987) Las especies de Adesmia de la serie Microphyllae (Leguminosae-Papilionoideae). Darwiniana (Argentina) 27: 315-388.

ULIBARRI E A \& A BURKART (2000) Sinopsis de las especies de Adesmia (Leguminosae, Adesmieae) de la Argentina. Darwiniana (Argentina) 38: 59-126.

VAN DER HAMMEN T \& A CLEEF (1983) Datos para la historia de la flora andina. Revista Chilena de Historia Natural 56: 97-107.

VILLAGRÁN C \& LF HINOJOSA (1997) Historia de los bosques del sur de Sudamérica, II: análisis fitogeográfico. Revista Chilena de Historia Natural 70: 241- 267.

YOUNG K, C ULLOA, J LUTEYN \& S KNAPP (2002) Plant evolution and endemism in Andean South America: An introduction. The Botanical Review 68: $4-21$ 
ZULOAGA FO \& O MORRONE (1999) Adesmia DC. En: Zuloaga FO \& O Morrone (eds) Catálogo de las plantas vasculares de la Argentina. Dicotyledoneae: 627- 639. Monograph in Systematic Botany II from The Missouri Botanical Garden Press, Saint Louis, Missouri, USA.

ZUNINO M (2000) El concepto de área de distribución: algunas reflexiones teóricas. En: Martín-Piera Morrone FJJ \& A Melic (eds) Hacia un proyecto
CYTED para el inventario y estimación de la diversidad entomológica en Iberoamérica: PrIBES 2000: 77-85. m3m-. Monografías Tercer Milenio, volumen 1. Sociedad Entomológica Aragonesa (SEA), Zaragoza, España.

ZUNINO M \& A ZULLINI (2003) Biogeografía. La dimensión espacial de la evolución. Fondo de Cultura Económica, México, Distrito Federal, México. 359 pp.

Editor Asociado: Thomas Kitzberger

Recibido el 8 de septiembre de 2005; aceptado el 29 de marzo de 2006

\section{ANEXO 1A}

Matriz utilizada en el análisis de parsimonia de endemismos de cuadrículas de $1^{\circ}$ latitud $\mathrm{x} 1^{\circ}$ longitud, 135 cuadrículas x 22 trazos individuales

Matrix used in parsimony analysis of endemicity, grids of $1^{\circ}$ latitude x $1^{\circ}$ longitude, 135 grids x 22 individual tracks

\begin{tabular}{ll}
\hline Out & 0000000000000000000000 \\
\hline 440 & 0000000001000000000000 \\
476 & 0000000000000000000100 \\
477 & 0000000000000000000100 \\
478 & 0000000000000000000100 \\
479 & 0000000001000000000100 \\
480 & 0000000001000000000100 \\
481 & 0000000000000000000100 \\
516 & 0000001000000000000000 \\
517 & 0000001000000000000100 \\
518 & 0000000001000000000100 \\
553 & 0000001000000000000000 \\
554 & 0000001001000000000100 \\
555 & 0000000001000000000100 \\
556 & 0000000001000000000000 \\
557 & 0000000001000000000000 \\
589 & 0000001000000000000000 \\
590 & 0000001000000000000100 \\
592 & 0000000001000000000000 \\
593 & 0000000001000000000000 \\
623 & 0000001000000000000100 \\
626 & 0000000001000000000100 \\
655 & 0000001000000000000100 \\
656 & 0000001000000000000100 \\
657 & 0000000000000000000100 \\
658 & 0000000001000000000100 \\
687 & 0000001000000000000000 \\
688 & 0000001000000010000100 \\
689 & 0000000000000000000100 \\
690 & 0000100001000000000100 \\
720 & 0000100000000010000100 \\
721 & 0000100000000010000100 \\
722 & 0000100001000000000000 \\
723 & 0000000000000001000000 \\
724 & 0000000000000001000000 \\
750 & 0010000000000000000000 \\
751 & 0010000000000000000000 \\
752 & 0000100000000010000100 \\
753 & 0000100001000000000100 \\
754 & 0000100001000001000000 \\
781 & 0010000000000000000000 \\
782 & 0010000000000000000000 \\
783 & 0010100000000000000100 \\
784 & 0010100000000000000100 \\
785 & 0010000000000001000100 \\
809 & 0000000000000000100000 \\
&
\end{tabular}

\begin{tabular}{cc}
\hline Out & 0000000000000000000000 \\
\hline 811 & 0010010000000000000000 \\
812 & 0010110000000000000100 \\
813 & 0010100000000000000000 \\
834 & 0000000000000000100000 \\
836 & 0010110000000000000000 \\
837 & 0010100000000000000000 \\
858 & 0000000000000000100000 \\
860 & 1010110000000000000000 \\
861 & 0010100000000000000000 \\
882 & 0000000000000000100000 \\
883 & 0000010000000000000000 \\
884 & 1010110000000000000010 \\
885 & 0010100000000000000000 \\
906 & 0000000000000000100000 \\
907 & 1000110000000000000000 \\
908 & 1010100000000000000010 \\
930 & 1000010000000000100000 \\
931 & 1000110000000000000010 \\
932 & 0000100000000000000010 \\
952 & 0100000000000000100000 \\
953 & 1100010000000000100000 \\
954 & 1000100000000100000010 \\
955 & 0000100000000000000000 \\
975 & 0000000010000000000000 \\
976 & 1100000010000000000000 \\
977 & 1000100000000100000010 \\
998 & 0100000010000000000000 \\
999 & 1000100000000100010010 \\
1000 & 0000100000000000000000 \\
1018 & 0000000010000000000000 \\
1019 & 0100000010011000000000 \\
1020 & 0001000100101100011000 \\
1039 & 0101000000010000011000 \\
1040 & 0001000100111000011000 \\
1059 & 0001000000010000000000 \\
1060 & 0000000100111000011001 \\
1076 & 0001000000110000001000 \\
1077 & 0001000000100000001001 \\
1094 & 0000000000110000001000 \\
1095 & 0000000000000000001001 \\
1112 & 0000000000110000001001 \\
1113 & 0000000000100000000001 \\
1128 & 0000000000000000000001 \\
1129 & 0000000000110000001001 \\
1130 & 0000000000000000000001 \\
&
\end{tabular}

\begin{tabular}{ll}
\hline Out & 0000000000000000000000 \\
\hline 1142 & 0000000000010000000001 \\
1143 & 0000000000110000001001 \\
1154 & 0000000000000000000001 \\
1155 & 0000000000100000001001 \\
1156 & 0000000000000000000001 \\
1167 & 0000000000100000001001 \\
1168 & 0000000000100000000001 \\
1179 & 0000000000100000001001 \\
1180 & 0000000000100000000001 \\
1188 & 0000000000100000001001 \\
1189 & 0000000000100000000001 \\
1190 & 0000000000100000000001 \\
1191 & 0000000000100000000000 \\
1192 & 0000000000000000001000 \\
1198 & 0000000000000000001000 \\
1199 & 0000000000000000001000 \\
1200 & 0000000000000000001001 \\
1201 & 0000000000100000001001 \\
1202 & 0000000000000000001000 \\
1206 & 0000000000000000000001 \\
1207 & 0000000000000000000001 \\
1211 & 0000000000100000000001 \\
1212 & 0000000000100000000000 \\
1216 & 0000000000000000000001 \\
1217 & 0000000000000000000001 \\
1219 & 0000000000000000000001 \\
1220 & 0000000000000000000001 \\
1221 & 0000000000100000000000 \\
1222 & 0000000000100000000000 \\
1224 & 0000000000000000000001 \\
1225 & 0000000000000000000001 \\
1226 & 0000000000000000000001 \\
1227 & 0000000000000000000001 \\
1228 & 0000000000000000000001 \\
1229 & 0000000000000000000001 \\
1230 & 0000000000000000000001 \\
1235 & 0000000000000000000001 \\
1236 & 0000000000000000000001 \\
1237 & 0000000000000000000001 \\
1238 & 0000000000000000000001 \\
1245 & 0000000000000000000001 \\
1246 & 0000000000000000000001 \\
1252 & 0000000000000000000001 \\
1253 & 0000000000000000000001 \\
&
\end{tabular}




\section{ANEXO 1B}

Matriz utilizada en el análisis de parsimonia de endemismos de $1^{\circ}$ de longitud, 12 cuadrículas x 22 trazos individuales

Matrix used in parsimony analysis of endemicity, grids of $1^{\circ}$ longitude, 12 grids x 22 individual tracks

\begin{tabular}{ll}
\hline Out & 0000000000000000000000 \\
\hline 8 & 0000000000000000000100 \\
9 & 0000000000000000000101 \\
10 & 0000001000000000000101 \\
11 & 0100001011010000100101
\end{tabular}

\begin{tabular}{ll}
\hline Out & 0000000000000000000000 \\
\hline 12 & 1101011011111000111101 \\
13 & 1011111011110001111 \\
14 & 1010111001100010001111 \\
15 & 0010110001100010001111
\end{tabular}

\begin{tabular}{cc}
\hline Out & 0000000000000000000000 \\
\hline 16 & 0010100001100000001100 \\
17 & 0010100001100001001100 \\
18 & 0000000000000001000000
\end{tabular}

\section{ANEXO 1C}

Matriz utilizada en el análisis de parsimonia de endemismos de cuadrículas de $1^{\circ}$ de latitud, 40 cuadrículas x 22 trazos individuales

Matrix used in parsimony analysis of endemicity, grids of $1^{\circ}$ latitude, 40 grids x 22 individual tracks

\begin{tabular}{ll}
\hline Out & 0000000000000000000000 \\
\hline 10 & 0000000001000000000000 \\
11 & 0000000001000000000100 \\
12 & 0000001001000000000100 \\
13 & 0000001001000000000100 \\
14 & 0000001001000000000100 \\
15 & 0000001001000000000100 \\
16 & 0000001001000000000100 \\
17 & 0000101001000010000100 \\
18 & 0000100001000011000100 \\
19 & 0010100001000011000100 \\
20 & 0010100000000001000100 \\
21 & 0010110000000000100100 \\
22 & 0010110000000000100000
\end{tabular}

\begin{tabular}{ll}
\hline Out & 0000000000000000000000 \\
\hline 23 & 1010110000000000100000 \\
24 & 1010110000000000100010 \\
25 & 1010110000000000100010 \\
26 & 1000110000000000100010 \\
27 & 1100110000000100100010 \\
28 & 1100100010000100000010 \\
29 & 1100100010000100010010 \\
30 & 0101000110111100011000 \\
31 & 0101000100111000011000 \\
32 & 0001000100111000001001 \\
33 & 0001000000110000001001 \\
34 & 0000000000110000001001 \\
35 & 0000000000110000001001
\end{tabular}

\begin{tabular}{ll}
\hline Out & 0000000000000000000000 \\
\hline 36 & 0000000000110000001001 \\
37 & 0000000000110000001001 \\
38 & 0000000000100000001001 \\
39 & 0000000000100000001001 \\
40 & 0000000000100000001001 \\
41 & 0000000000100000001001 \\
42 & 0000000000100000001001 \\
43 & 0000000000100000000001 \\
44 & 0000000000100000000001 \\
45 & 0000000000000000000001 \\
46 & 0000000000000000000001 \\
47 & 0000000000000000000001 \\
48 & 0000000000000000000001
\end{tabular}

